Article

\title{
Design and Performance Evaluation of Multi-Gb/s Silicon Photonics Transmitters for High Energy Physics ${ }^{\dagger}$
}

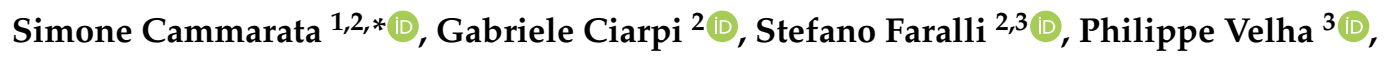 \\ Guido Magazzù ${ }^{2}$, Fabrizio Palla ${ }^{2}$ (i) and Sergio Saponara ${ }^{1}$ (1) \\ 1 Dipartimento Ingegneria dell'Informazione, Università di Pisa, Via G. Caruso 16, 56122 Pisa, Italy; \\ sergio.saponara@unipi.it \\ 2 Istituto Nazionale di Fisica Nucleare, Sezione di Pisa, L. Pontecorvo 3, 56127 Pisa, Italy; \\ gabriele.ciarpi@ing.unipi.it (G.C.); stefano.faralli@santannapisa.it (S.F.); \\ Guido.Magazzu@pi.infn.it (G.M.); Fabrizio.Palla@cern.ch (F.P.) \\ 3 Scuola Superiore Sant'Anna, Istituto TeCIP, Via G. Moruzzi 1, 56124 Pisa, Italy; \\ philippe.velha@santannapisa.it \\ * Correspondence: simone.cammarata@phd.unipi.it; Tel.: +39-050-2217505 \\ + This paper is an extended version of our paper published in ApplePies 2019: Applications in Electronics \\ Pervading Industry, Environment and Society, Pisa, Italy, 11-13 September 2019; pp. 11-18.
}

Received: 15 June 2020; Accepted: 8 July 2020; Published: 10 July 2020

\begin{abstract}
Optical links are rapidly becoming pervasive in the readout chains of particle physics detector systems. Silicon photonics ( $\mathrm{SiPh}$ ) stands as an attractive candidate to sustain the radiation levels foreseen in the next-generation experiments, while guaranteeing, at the same time, multi-Gb/s and energy-efficient data transmission. Integrated electronic drivers are needed to enable SiPh modulators' deployment in compact on-detector front-end modules. A current-mode logic-based driver harnessing a pseudo-differential output stage is proposed in this work to drive different types of SiPh devices by means of the same circuit topology. The proposed driver, realized in a $65 \mathrm{~nm}$ bulk technology and already tested to behave properly up to an $8 \mathrm{MGy}$ total ionizing dose, is hybridly integrated in this work with a lumped-element Mach-Zehnder modulator (MZM) and a ring modulator (RM), both fabricated in a $130 \mathrm{~nm}$ silicon-on-insulator (SOI) process. Bit-error-rate (BER) performances confirm the applicability of the selected architecture to either differential and single-ended loads. A $5 \mathrm{~Gb} / \mathrm{s}$ data rate, in line with the current high energy physics requirements, is achieved in the RM case, while a packaging-related performance degradation is captured in the MZM-based system, confirming the importance of interconnection modeling.
\end{abstract}

Keywords: silicon photonics; optical communications; Mach-Zehnder modulator; ring modulator; current-mode logic; CMOS; pseudo-differential; radiation hardness; high energy physics; BER characterization

\section{Introduction}

Multi-Gb/s serial data links are largely deployed in high energy physics (HEP) environments to exchange data between on-detector readout modules and off-site processing electronics. Luminosity and sensors' granularity growth foreseen for future HEP experiments will lead to a tremendous increase in data volumes and radiation exposures [1]. State-of-the-art radiation-tolerant optical links have been developed in the framework of versatile link (VL) and gigabit transceiver (GBT) projects and are now deployed inside the Large Hadron Collider (LHC) experiments at CERN [2,3]. They are currently based on both single-mode edge-emitting lasers (EELs) and multi-mode vertical cavity surface emitting 
lasers (VCSELs) [4], which are directly modulated by means of radiation-hard application-specific integrated circuits (ASICs) [5]. Laser diode-based technologies however suffer from radiation-induced degradation caused by displacement damage. In the next-generation HEP apparatus, particle fluences will become too high $\left(\approx 10^{16} \mathrm{~cm}^{-2} 1 \mathrm{MeV}\right.$ neutron equivalent) to guarantee VCSELs' operation in the innermost detector layers, and suitable alternatives need to be investigated [6].

In the last few decades, silicon photonics ( $\mathrm{SiPh}$ ) has attracted interest as a viable technology for the realization of power-efficient short-reach optical interconnects. The low-cost, high-yield, and complementary metal oxide semiconductor (CMOS) fabrication processes along with the possibility to integrate different optical functionalities on a single chip allowed it to rapidly become the workhorse of datacom businesses [7]. A similar traction is gradually rising also inside the HEP community since silicon ( $\mathrm{Si}$ ) has already been proven to be an excellent radiation-tolerant material [8]. Moreover, the technological compatibility with electronics opens up new levels of integration at the detector-level, which can alleviate material budget constraints [9].

Much effort has been focused on verifying whether SiPh-based data links can tolerate the harsh conditions typical of HEP experiments. SiPh devices are relatively insensitive to neutron radiation, but they show strong degradation under ionizing X-rays exposure [10]. It was proven that the X-ray-induced impairments can be moved beyond the MGy-level by following some radiation-hardening-by-design (RHBD) techniques [11] or by periodically modifying the driving conditions [12].

Given the recent technology readiness level (TRL) boost, SiPh modulators are starting to become almost as commercial off-the-shelf (COTS) components [13]. Many foundries already provide design kits and layout cells of photonic components that ever more frequently lead to modulation performances up to $50 \mathrm{~Gb} / \mathrm{s}$ [14]. Nevertheless, equally high-speed and radiation-resistant ASICs are needed to interface these photonic devices with the front-end electronics and make the complete readout units fit into compact modules. From a system-level viewpoint, the best achievement would be the realization of an electronic circuit that is capable of driving a broad variety of SiPh devices, mimicking the GBT driving flexibility for the laser diode case [15]. SiPh-based wavelength division multiplexing (WDM) schemes applied to HEP scenarios are currently under development, and the exploitation of general-purpose drivers could indeed help to attain high aggregate bandwidth readout systems [16].

In this work, we will show that small architectural changes to conventional CMOS drivers for $\mathrm{SiPh}$ devices can enable multi-Gb/s transmission on both lumped-element Mach-Zehnder modulators (MZMs) and ring modulators (RMs), at least for relatively low bandwidths compared to datacom frameworks, but still of interest for HEP applications. This paper extends the contribution in [17] in terms of conceptual modeling and experimental results, evaluating the operation of a full-custom CMOS driver with different classes of SiPh modulators.

Section 2 provides a background on SiPh modulators and traditional driver circuits. Section 3 discusses the proposed driver topology and describes the circuital choices that make it appropriate both for MZMs, as well as RMs. Finally, experimental procedures and system-level characterization results are respectively presented in Sections 4 and 5. Conclusions and further perspectives are drawn in Section 6.

\section{Background}

\subsection{Silicon Photonics Modulators}

In SiPh technologies, an optical modulator is usually realized by embedding a pn-junction inside a waveguide [18]. It is well known that the presence of free charge carriers, i.e., electrons and holes, can change the refractive index of a semiconductor material [19]. By varying the pn-junction bias voltage, free-carriers' distributions change across the waveguide, and the phase velocity of the propagating optical mode is modified. Depletion-mode operation ensures high-speed phase 
modulation since only majority-carriers' dynamics are involved [20]. Amplitude modulation can be achieved by inserting phase shifters into interferometric, e.g., MZMs [21], or resonant architectures, e.g., RMs [22,23]. Information is indeed typically encoded in the light intensity, and the simple on-off keying $(\mathrm{OOK})$ is one of the most adopted modulation formats, although more complex schemes are emerging, e.g., PAM-4 [24].

In order to make efficient designs for the electronic driver, it is important to carry out an electrical equivalent circuit of the phase shifter under study. The reverse-biased diode can be represented by a voltage-dependent junction capacitance in series with the access resistances associated to the ohmic conduction through the anode and cathode doped slabs. The junction capacitance per phase shifter length $C_{l}$ is typically around hundreds of $\mathrm{fF} / \mathrm{mm}$, while the per-unit-length resistance $R_{l}$ is normally about a few $\Omega \cdot \mathrm{mm}$. For modulation wavelengths $\lambda_{\text {rf }}$, which are greatly larger than the device dimensions, i.e., $l \ll \lambda_{\text {rf }}$, the appropriate circuit model for a phase shifter of length $l$ is depicted in Figure 1a. In the limits of this approximation, the overall device behavior is captured by means of lumped-elements (LEs), and the intrinsic $(-3 \mathrm{~dB})$ bandwidth results [20]:

$$
f_{3 \mathrm{~dB}}=\frac{1}{2 \pi\left(R_{l} C_{l}+Z_{\mathrm{drv}} l C_{l}\right)}
$$

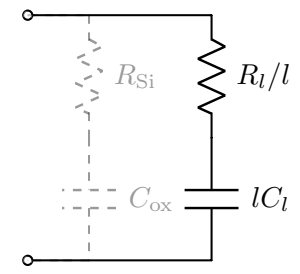

(a) Lumped-element.

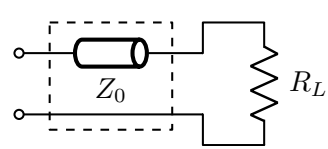

(b) Terminated traveling-wave.

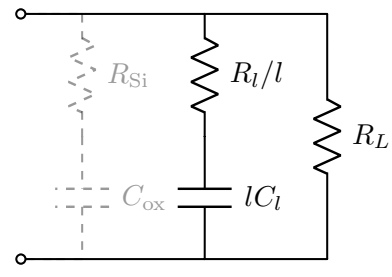

(c) Terminated lumped-element (TW device for large $\lambda_{\text {rf }}$ wavelengths).

Figure 1. Equivalent circuit models for silicon photonics ( $\mathrm{SiPh}$ ) phase shifters. Gray-colored elements $\left(R_{\mathrm{Si}}, C_{\mathrm{ox}}\right)$ represent signal coupling to the substrate via the buried oxide of silicon-on-insulator (SOI) wafers. They can be extracted by fitting S-parameters on-wafer measurements and then considered for the design [25]. In the TW case, the characteristic impedance $Z_{0}$ already takes into account the contribution of all the layers surrounding the on-chip transmission line.

Assuming a purely real driver output impedance $Z_{\mathrm{drv}}$ and neglecting all the parasitic contributions. Being proportional to the amount of charge carriers that are swept in and out the active device region, $C_{l}$ is directly related to the phase shifting efficiency, which is commonly quantified by the $V_{\pi} l$ figure of merit. Since the free-carrier effects are weak in silicon, $V_{\pi} l$ typically ranges between $10 \mathrm{~V} \cdot \mathrm{mm}$ and even $40 \mathrm{~V} \cdot \mathrm{mm}$ depending on the implant conditions [26]. Because the available voltage span is typically no more than a few volts in deep sub- $\mu$ m CMOS technologies, phase shifters are normally operated with voltage swings substantially lower than the rated $V_{\pi}$ [27]. Interference-based modulators are then made a few millimeters long to accumulate enough phase shift and provide reasonable optical extinction ratios (ERs). As a consequence, the total capacitance $l C_{l}$ is enlarged, and $f_{3 \mathrm{~dB}}$ is diminished according to Equation (1). On the other hand, compact devices, such as RMs, present small lumped capacitances and can guarantee high cutoff frequencies from a purely electrical point of view, i.e., ignoring photon lifetime-related effects.

To cope with the capacitance-limited response of long modulators, traveling-wave (TW) devices are usually conceived. By designing phase shifters as transmission lines (TLs), the capacitive loading could be accurately "mixed up" with the inductive contribution of the coplanar electrodes, and the whole device, when properly terminated, globally appears as a (resistive) load equal to the TL's characteristic impedance $Z_{0}$ (Figure $1 \mathrm{~b}$ ). By choosing $R_{L} \approx Z_{0} \approx Z_{\mathrm{drv}}$, impedance matching can be theoretically achieved (neglecting parasitics), and by making them equal to $50 \Omega$, the compatibility with common testing instrumentation can be maintained. However, the TW behavior manifests only at 
frequencies for which the LE approximation no longer holds. Otherwise, for relatively low modulation frequencies, a TW modulator appears as an LE device in parallel with the termination impedance $R_{L}$, as sketched in Figure 1c.

\subsection{Driving Architectures}

Depending on the SiPh modulator to be driven, some circuit architectures are more suitable than others, in particular regarding the output stage. As a general frame of reference, differential loads are usually driven by current mode logic (CML)-inspired architectures, while single-ended (SE) devices are traditionally actuated with CMOS inverter-like stages. The latter indeed consume less static power, but are characterized by lower speed ratings compared to their current-steering counterparts [26]. Moreover, TW loads need to be as closely impedance-matched as possible with the conditioning electronics to avoid signal reflections, while LE modulators require low driving impedances $\left(Z_{\mathrm{drv}}\right)$ to extend the operational bandwidth, according to Equation (1).

MZMs have a differential structure that is always actuated in push-pull (PP), i.e., by applying opposite signals to the phase shifters in the interferometer arms. Standard common-source (CS) differential pairs are generally implemented to realize PP driving. Figure 2 reports two widely exploited topologies for TW-MZMs [28]. In the "open-drain" version, all the available current flows in the modulator without undergoing partitionings, so the maximum available voltage swing develops on the termination resistors. This notwithstanding, the high output impedance provided by the drain connections makes this architecture vulnerable to impedance discontinuities. Both near-end and far-end reflections are usually dealt with using a "double-termination" structure [29], which resembles a standard CML buffer where the biasing load branches are impedance-matched to the SiPh TW load. However, in this case, the modulator is fed with a lower voltage swing compared to the "open-drain" version, in the same power consumption conditions. The internal termination is indeed connected in parallel to the SiPh modulator, thus lowering the effective load impedance.

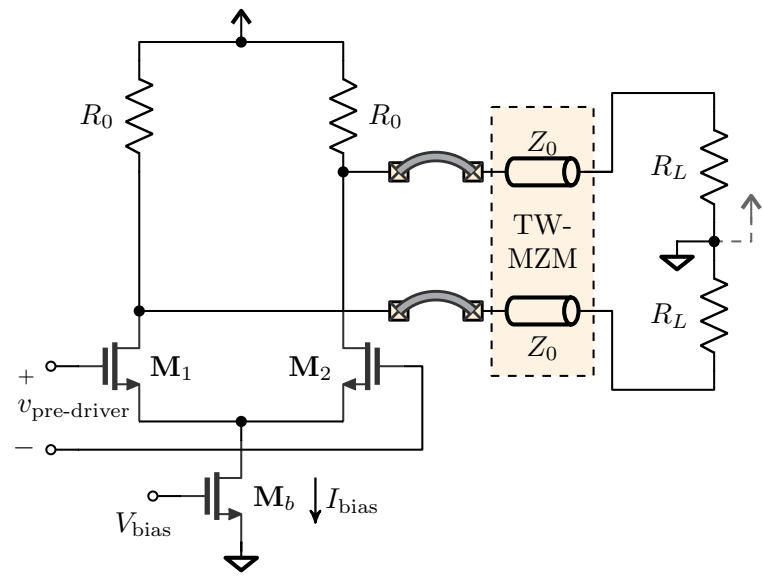

(a)

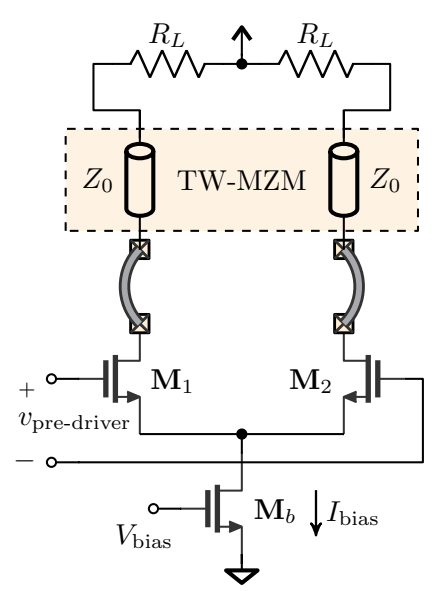

(b)

Figure 2. Output stages suitable for TW device driving. (a) "Double-termination" current-steering common-source pair. Mach-Zehnder modulator (MZM) arm terminations could be either grounded or tied to supply voltage (dashed line). (b) “Open-drain” current-steering common-source pair.

Differential LE modulators, e.g., sections of segmented MZMs, can also be actuated by current-steering stages. As sketched in Figure 3a, source follower (SF) output stages can be used in this case to present a low output impedance [30]. On the other hand, drivers for single-ended LE phase shifters are traditionally designed by stacking several transistors on top of each other in a CMOS configuration to extend the output voltage swing, as shown in Figure 3b. Floating supply rails, together with level shifter circuits, are then harnessed to meet technological reliability requirements [31]. In the specific case of RMs, two different output stages are sometimes used to drive the modulator terminals 
independently [32]. In this way, a little forward bias can be introduced into the driving waveform to enlarge the optical ER.

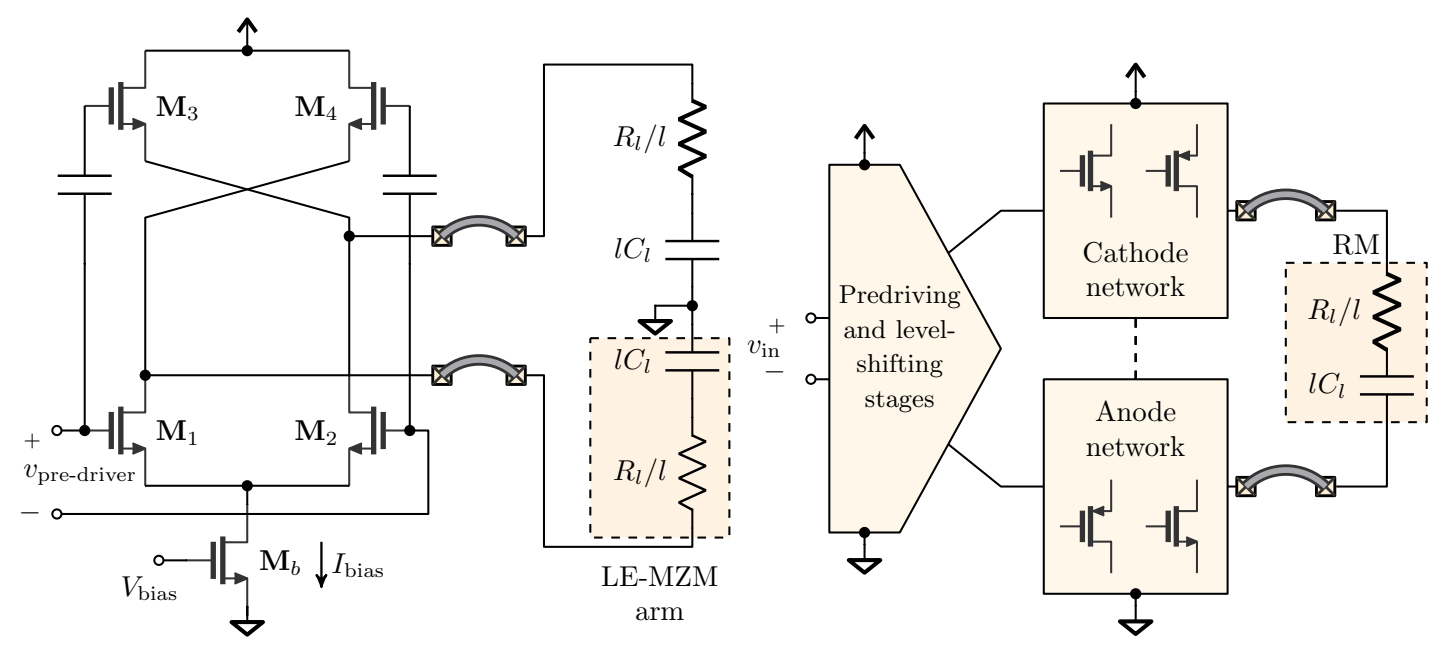

(a) Source follower (SF)-based

(b) CMOS inverter-like.

current-steering.

Figure 3. Output stages suitable for lumped-element (LE) device driving. (a) Source follower-based current-steering pair. (b) CMOS gate-like topology. Different transistor stacking arrangements can be exploited with the aid of floating supply rails [26,31,33-35]. Dealing with ring modulators (RMs), cathode and anode driver stages are sometimes separated (dashed line) to provide a slight forward bias to the pn-junction [32].

\section{Proposed Driver Design}

Radiation tolerance requirements pose severe limitations in the design of an ASIC. Recently, several studies were conducted on deep sub- $\mu \mathrm{m}$ platforms suitable for high-speed applications, e.g., $65 \mathrm{~nm}$ [36] and, even more recently, $28 \mathrm{~nm}$ [37] technologies. Total ionizing dose (TID)-induced charge trapping determines transistor parameter drifts and leads to the formation of current leakage paths in active regions next to shallow trench isolations (STIs) [38]. Moreover, p-type MOSFETs result in being less tolerant than n-type ones, and radiation-induced short/narrow channel effects are reported. As a consequence, few RHBD techniques have been drawn to extend the radiation hardness of a given ASIC design [39]. According to those rules, p-type and minimum-length MOSFETs have not been used throughout the design, and only thin-oxide enclose layout transistors (ELTs) have been exploited [40].

The whole driver consists of a five stage tapered chain of standard CML buffers followed by the proposed output stage, which needs to be capable of driving both differential, as well as single-ended $\mathrm{SiPh}$ modulators. The aforementioned radiation-related guidelines affect the architecture choice for the last stage. CMOS-like solution are surely not feasible since they harness p-MOSFETs. However, current steering structures, such as those presented in Section 2.2, are also not suitable. Connecting an LE phase shifter directly to the two output leads, the differential swinging would determine the pn-junction forward biasing. Instead, loading only one branch with a single-ended load would unbalance the circuit. Dummy loads can in principle be used to restore the balance, as is sometimes done in certain drivers [41]. Nevertheless, they undoubtedly introduce mismatches and possibly lead to spurious phase modulation at GHz-range frequencies, affecting jitter performances [29].

As shown in Figure 4, a pseudo-differential structure, i.e., a differential pair without a tail current source, appears to be a viable solution for the problem. Resistive pull-up is preferred to the SF-based topologies to save the voltage drop on the upper transistors and get the highest possible swing out of the circuit. Conventional SF-based topologies do not produce voltage gain, and their usage leads to more complex pre-driving and output stage designs [42]. For the ease of testing and to drive also terminated devices, the internal load resistors, indicated with $R_{0}$ in Figure 4 , are nominally chosen 
as $50 \Omega$. The tail current source removal also helps in enlarging the peak-to-peak output swing by saving the voltage headroom normally left for the biasing MOSFET (on the order of an overdrive voltage). The common-mode level of the last pre-driving CML stage should be lowered approximately by the same amount in order to drive the input transistors of the output stage properly. Anyway, the intrinsic input common-mode sensitivity associated with pseudo-differential structures has no great importance in this specific case since, dealing with non-return-to-zero (NRZ) signals, the circuit operates in switching large-signal mode [29]. To further increase the output swing, a doubled supply voltage $V_{\mathrm{DD}_{\mathrm{H}}}=2.4 \mathrm{~V}$ (with respect to the $1.2 \mathrm{~V}$ applied elsewhere) is used in the last stage. A cascode structure is then implemented to keep voltage stresses on MOSFETs in the technology's safe operating area (SOA). Harnessing thick-oxide devices in the series transistor stacking could have allowed increasing even more the output supply voltage. However, radiation-hardening constraints prevented their exploitation for circuits targeted to sustain MGy-range TIDs.

Inductive peaking was also introduced in the last two stages to extend the bandwidth [43]. Inductors $\left(L_{p}\right)$ have been realized with a differential layout with a central tap to save area. Further details about the circuit design and purely electrical measurement results can be found in [44].

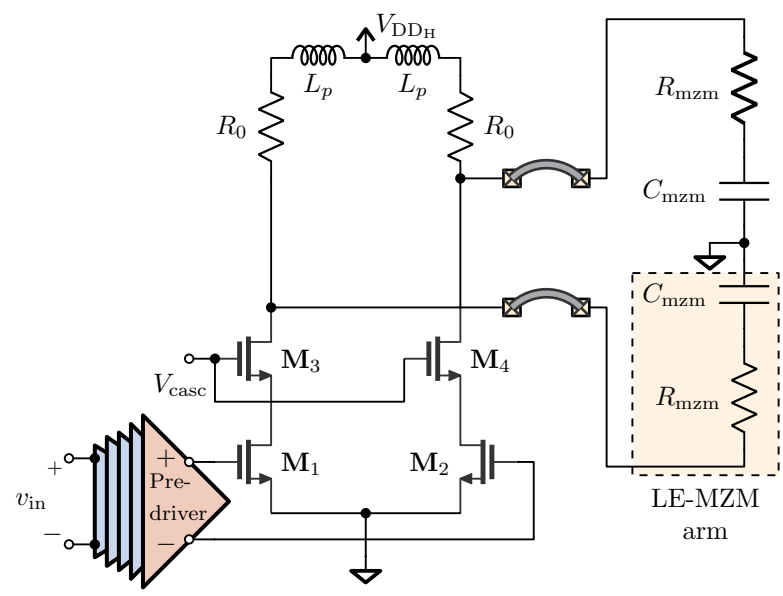

(a)

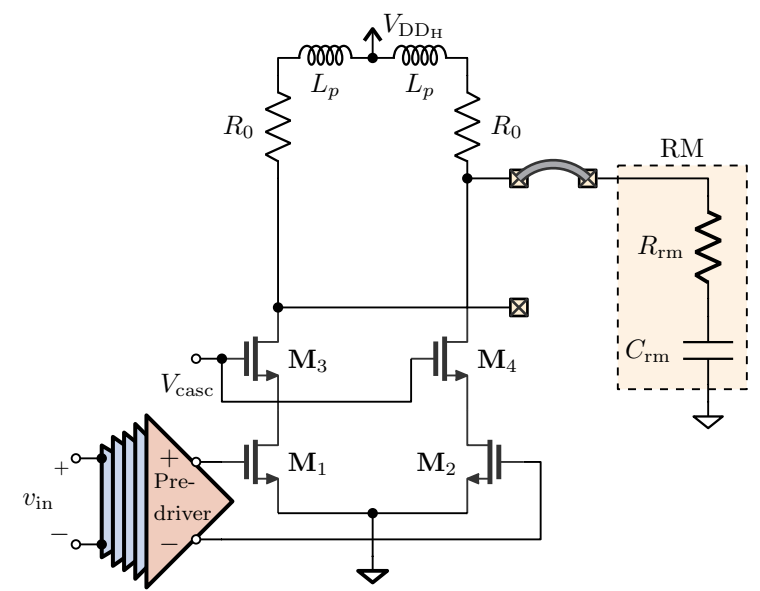

(b)

Figure 4. Proposed pseudo-differential output stage applied to LE-MZMs (a) and RMs (b). The five stage tapered chain of current mode logic $(C M L)$ buffers is also indicated, with the last pre-driving stage (colored differently), which is inductively peaked.

\section{Experimental Setup}

\subsection{Samples}

The proposed driver was included in a $1 \mathrm{~mm} \times 1 \mathrm{~mm}$ electronic integrated circuit (EIC) fabricated in a commercial-grade radiation-hard $65 \mathrm{~nm}$ CMOS technology. The circuit radiation resistance was previously tested exposing the whole EIC to X-rays at the INFN-Padova facility. Proper operation of the standalone driver was observed till a total ionizing dose (TID) of $8 \mathrm{MGy}$, which was the highest dose level reached during the test because of limited testing time. A 30\% voltage amplitude degradation with respect to the pre-irradiation value was observed [17]. In addition, a single event upset (SEU) analysis of the same circuit was carried out in [44], proving its HEP-level radiation-tolerance. 
In this work, the proposed driver was tested together with two SiPh optical modulators fabricated in a SiPh $130 \mathrm{~nm}$ silicon-on-insulator (SOI) process, i.e., an MZM and an RM. They were based on the corresponding building blocks provided in the foundry's process design kit (PDK) and were laid out in a $5 \mathrm{~mm} \times 5 \mathrm{~mm}$ photonic integrated circuit (PIC) [45]. They both relied on $450 \mathrm{~nm} \times 220 \mathrm{~nm}$ rib-waveguide phase shifters with deep-etched slabs and used standard doping levels.

The MZM was $1 \mathrm{~mm}$ long and was drawn with unbalanced arms, i.e., one arm was physically made longer, which allowed tuning the operating point by changing the laser wavelength. Each arm was sized as a coplanar waveguide (CPW), and the electrodes' layout matched the output port pattern of the full-custom driver. Although having been designed as a TW device, each MZM arm lacked an on-chip termination and effectively behaved as a lumped-element for the frequencies of interest. The associated pn-junction capacitance $C_{\text {mzm }}$ was estimated to be around $0.5 \mathrm{pF}$. Wafer-level radio-frequency $(\mathrm{RF})$ measurements made on comparable MZM designs ensured that the electro-optical modulation bandwidth was above $10 \mathrm{GHz}$ with a proper termination [14].

The ring modulator had a $7.5 \mu \mathrm{m}$ radius resulting in a free spectral range (FSR) of about $13 \mathrm{~nm}$. As shown in Figure 5, the resonance next to $1.55 \mu \mathrm{m}$ had a full-width at half-maximum (FWHM) of about $240 \mathrm{pm}$ and an extinction depth of nearly $20 \mathrm{~dB}$. The spectrum was measured by feeding the device with a tunable external cavity laser (ECL) and sweeping the wavelength in the $1510 \mathrm{~nm}$ to $1570 \mathrm{~nm}$ range with a $0.82 \mathrm{pm}$ resolution. The LE capacitance $C_{\mathrm{rm}}$ associated with the $\mathrm{RM}$ was expected to be on the order of a few tens of femtofarads. Electronic and photonic dies were placed on a printed circuit board (PCB) and bonded with $40 \mu \mathrm{m}$ diameter aluminum (Al) bond-wires. Tapered coplanar transmission lines were realized on the PCB to maintain the $50 \Omega$ impedance environment while transitioning from the centimeter-scale footprint of the high-speed SMA (SubMiniature, Version A) connectors to the $100 \mu \mathrm{m}$ bond-pads' pitch. A PCB with the same layout was used for both characterizations. The complete packaged sample is shown in Figure 6.

Fiber-to-PIC light coupling was achieved by means of on-chip grating couplers and quasi-planar fiber-arrays. The latter were preferred to the vertical fiber couplers because of their lower encumbrance on the PIC surface [46]. Fiber arrays were manually pigtailed with the aid of micro-mechanical stages and active alignment tools. An index-matched epoxy cured with ultra-violet (UV) light was used to bond the silica fiber holder to the PIC in the position that gave the best insertion loss. Given the $\mu \mathrm{m}$-scale positioning tolerance of the grating-based coupling, the fiber-array ribbons were also glued on the PCB to limit the mechanical stresses on the fiber holder during manual handling (see Figure 6).

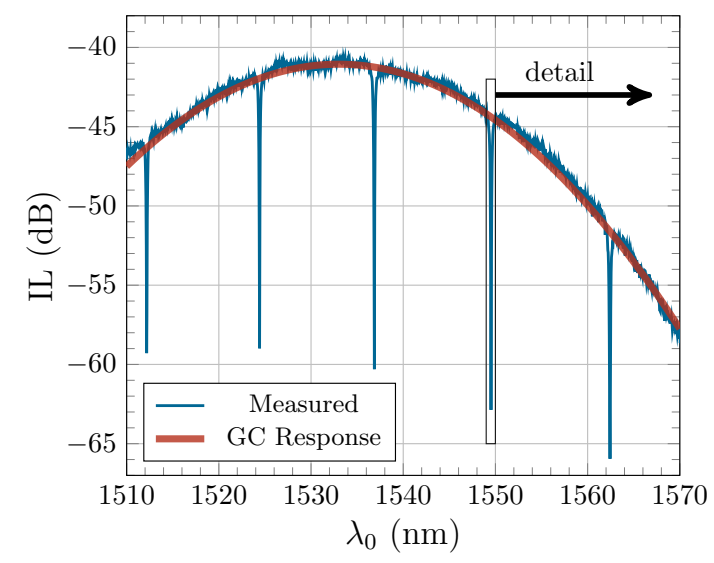

(a)

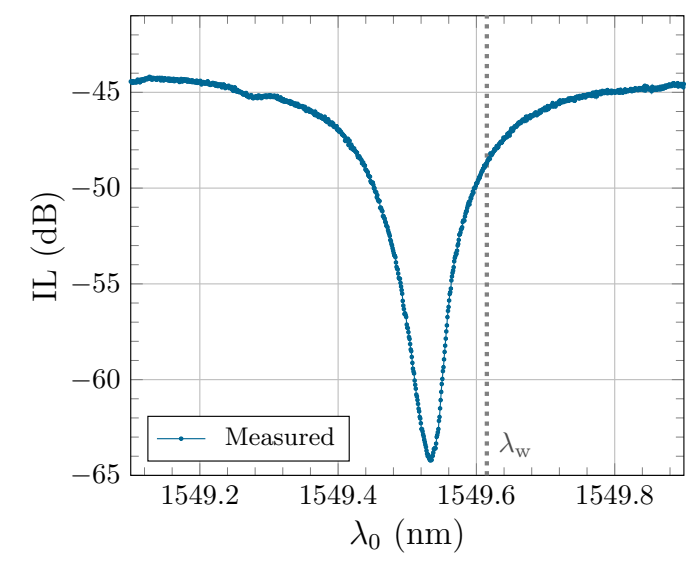

(b)

Figure 5. Spectral measurements made on the RM under test. (a) Complete spectrum. The grating coupler (GC) wavelength-dependent response is clearly visible. (b) Detail of the ring resonance used throughout the experimental characterization. IL, insertion loss; $\lambda_{0}$, optical wavelength. 


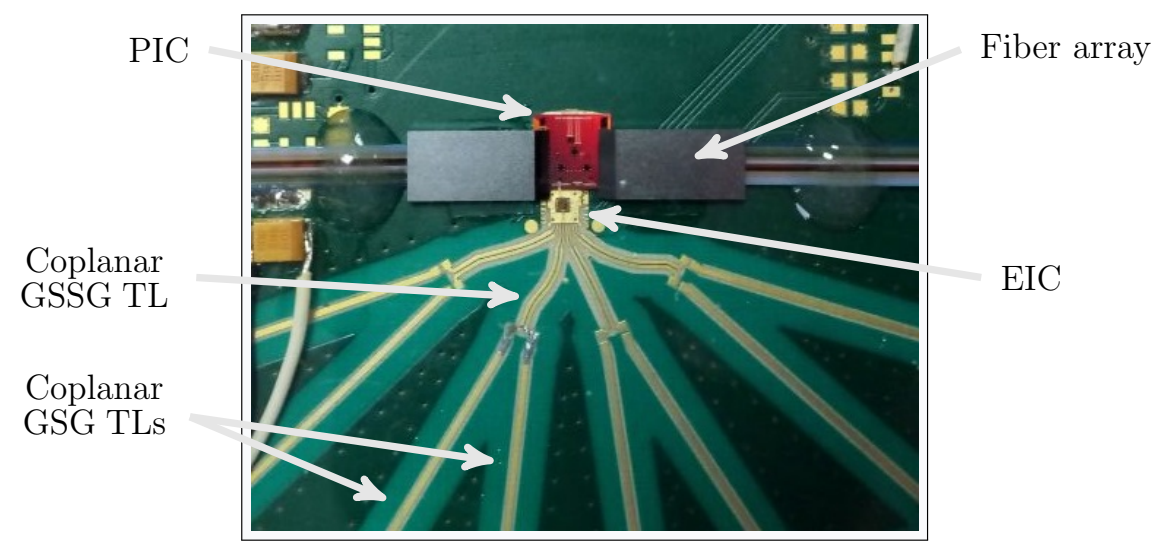

Figure 6. Structure of the complete packaged system. The sample appears the same in both case studies described in this work. They differ only for the SiPh modulator, which is attached to the electronic integrated circuit (EIC), i.e., MZM or RM. Coplanar transmission lines convey the input differential signal to the driver circuit. Starting with a ground-signal-ground (GSG) pattern from the SMA connectors, they merge into a single ground-signal-signal-ground (GSSG) line while approaching the EIC to match the bond-pads' pitch. TL, transmission line.

\subsection{Measurement Procedures}

A standard on-off keying (OOK) optical link was set up to characterize the SiPh-based transmitter with the devices under test. In order to quantify the transmission reliability, the bit error rate (BER) was chosen as a reference parameter. Optical links that were employed in HEP experiments covered at most distances on the order of hundreds of meters and, in principle, did not need to be optically amplified. To mimic the final application scenario, BER performances were evaluated at a constant optical signal-to-noise ratio (OSNR) by varying the optical power at the receiver [47].

Two different electronic dies were used for each SiPh modulator characterization. The measurements were made in distinct time slots; hence, the working conditions were not exactly the same for both cases. Figures 7 and 8 depict the experimental setups respectively built up for the MZM and the RM characterizations. Some elements were common to both scenarios. A $12.5 \mathrm{~Gb} / \mathrm{s}$ BER tester (BERT) stimulated the device under test (DUT) and calculated the BER. A pulse pattern generator (PPG) encoded a pseudo-random bit sequence (PRBS) on an NRZ electrical signal with voltage levels suitable to feed the input CML stage of the driver, i.e., a symmetric $400 \mathrm{mV}$ voltage swing with $1 \mathrm{~V}$ DC bias. A PRBS pattern $\left(2^{31}-1\right)$-long was chosen in order to make a repetitive measure with the largest number of bit combinations. The out-coupled modulated optical power was converted back in the electrical domain by means of a commercial $10 \mathrm{GHz}$-bandwidth module comprising a photo-detector (PD) and trans-impedance amplifier (TIA). An error detector (ED) compared the transmitter and received bit sequences and detected transmission errors. The two bit streams were synchronized by physically sharing the clock between PPG and ED. The voltage decision threshold and the sampling time on the ED were adjusted to get the lowest BER.

Optical power was provided by a tunable light source (TLS) near $1.55 \mu \mathrm{m}$. It was adjusted in order to operate the MZM in quadrature and the RM around the wavelength $\lambda_{w}$ shown in Figure 5, which was verified to generate the largest eye opening given the actual voltage swing delivered by the driver. Since grating couplers were only sensitive to transverse-electric (TE) fields, a polarization controller (PC) was placed upstream the DUT to maximize the coupled power. In the RM characterization, a 90/10 splitter was inserted before the PD in order to continuously monitor the output signal spectrum with an optical spectrum analyzer (OSA). This allowed measuring the OSNR at the receiver and detecting eventual working point shifts during measurements. Eye diagrams were captured by connecting the PD to a $40 \mathrm{GHz}$-bandwidth oscilloscope (the BERT clock signal was used to trigger the waveform acquisition). 


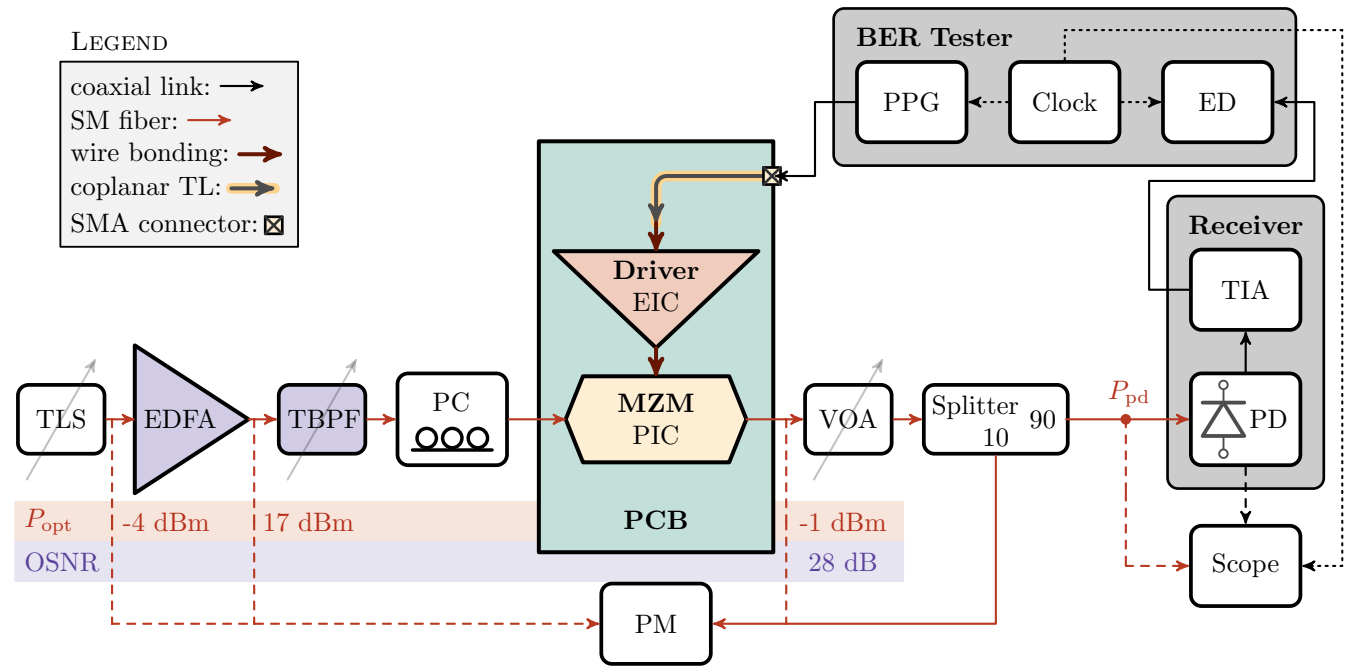

Figure 7. MZM characterization setup. $P_{\text {opt }}$ and $P_{\text {pd }}$ stand for the total power in the respective locations down the optical signal path. Dashed lines indicate connections that were done at some stages of the measurement sessions. TLS, tunable light source; EDFA, erbium-doped fiber amplifier; TBPF, tunable band-pass filter; PC, polarization controller; SM, single mode; VOA, variable optical attenuator; PM, power meter; PIC, photonic integrated circuit; EIC, electronic integrated circuit; PPG, pulse pattern generator; ED, error detector; TIA, trans-impedance amplifier; PD, photo-detector.

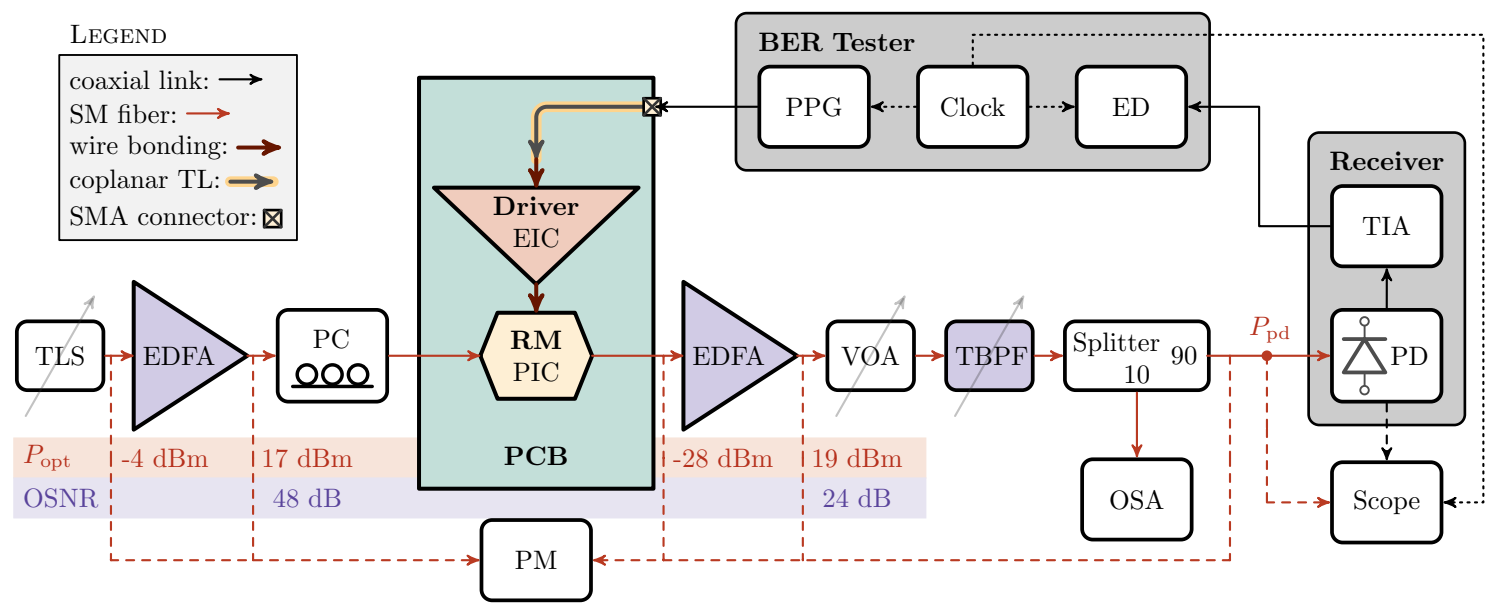

Figure 8. RM characterization setup. $P_{\text {opt }}$ and $P_{\text {pd }}$ stand for the total power in the respective locations down the optical signal path. Dashed lines indicate connections that were done at some stages of the measurement sessions. OSA, optical spectrum analyzer.

Both boards under test showed huge optical insertion losses (ILs), roughly $20 \mathrm{~dB}$ fiber-to-fiber IL for the MZM test chip and $40 \mathrm{~dB}$ in the RM case, as can be seen from Figure 5a. They were mainly attributed to accidental misalignments between the fiber array holder and the on-chip grating coupler array, probably occurred during the boards' shipping. Fiber-to-fiber ILs after the fiber array attachment were indeed measured to be around $12 \mathrm{~dB}$ in both cases. For this reason, some erbium-doped fiber amplifiers (EDFAs) were added to the optical signal chains. Two EDFAs were used for the RM testing because of the more severe optical losses in that specific case. A $0.3 \mathrm{~nm}$ tunable band-pass filter (TBPF) was also inserted in both setups to suppress the ASE-ASE (amplified spontaneous emission) beat noise in the photo-detection. These additional elements are highlighted in violet in Figures 7 and 8. EDFA gains and laser powers were tuned in order to have large OSNR values at the receiver and enable a system-level characterization even in the presence of this IL impairment. Large OSNR levels were necessary to attribute any transmission penalty to the transmitter functionality. 


\section{Results and Discussion}

Measured BERs as a function of the input power $P_{\mathrm{pd}}$ on the photo-detector are shown for different data rates in Figure 9 for the MZM testing case. The whole system worked up to a bit-rate of $1.5 \mathrm{~Gb} / \mathrm{s}$, while BER floors started to appear around $1.7 \mathrm{~Gb} / \mathrm{s}$, and a completely compromised transmission functionality was captured already at $2 \mathrm{~Gb} / \mathrm{s}$, as shown in Figure 10.
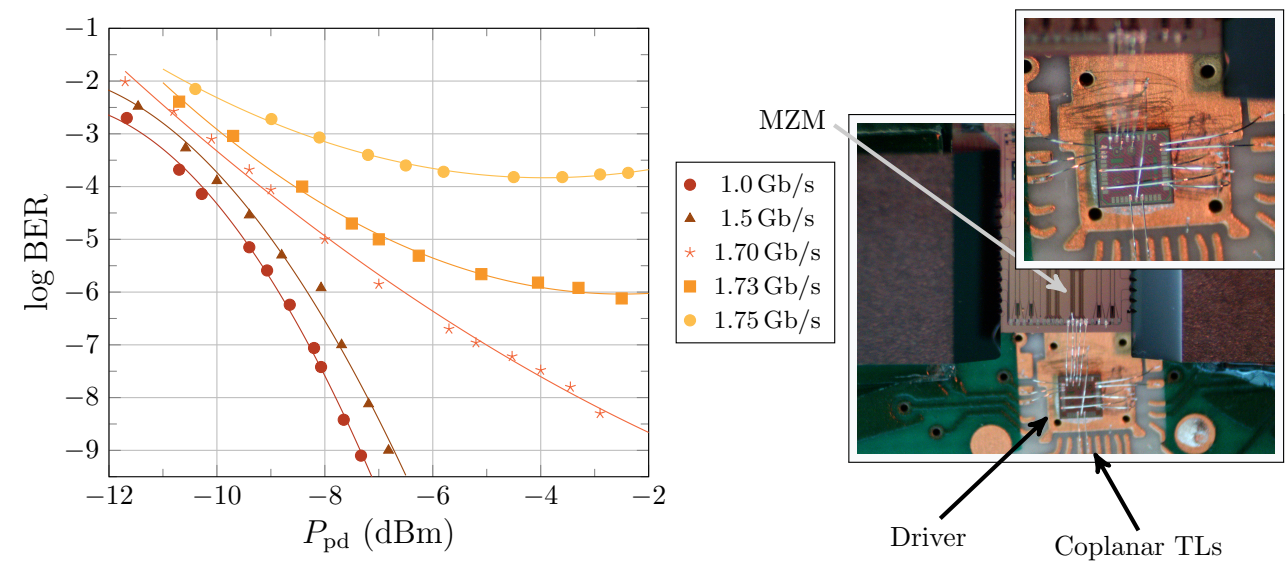

Figure 9. BER performances as a function of the received power $P_{\mathrm{pd}}$ for the MZM-based transmission system.

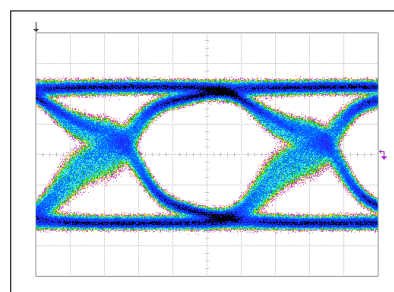

(a) $1.5 \mathrm{~Gb} / \mathrm{s}$

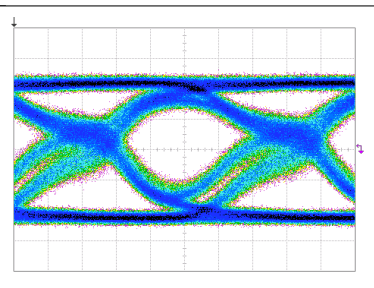

(b) $1.75 \mathrm{~Gb} / \mathrm{s}$

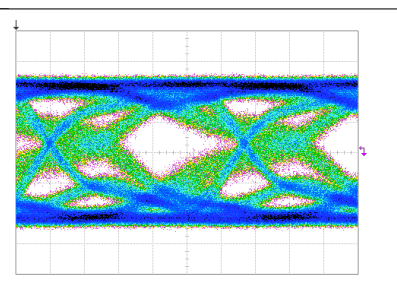

(c) $2 \mathrm{~Gb} / \mathrm{s}$

Figure 10. Eye diagrams on the PD output port in the MZM case study (scale $20 \mathrm{mV} /$ div).

The results for the RM-based system are instead shown in Figures 11 and 12. BER floors here became visible only above $5.5 \mathrm{~Gb} / \mathrm{s}$, and data transmission at $5 \mathrm{~Gb} / \mathrm{s}$ with BER below $10^{-9}$ was confirmed to be achievable with optical power levels on the receiver side $\left(P_{\mathrm{pd}}\right)$ greater than $-4 \mathrm{dBm}$. The eye diagrams in Figure 11 were all taken in the condition $P_{\mathrm{pd}}=-6 \mathrm{dBm}$ to be comparable. In that situation, the cases corresponding to the $4 \mathrm{~Gb} / \mathrm{s}$ and $5 \mathrm{~Gb} / \mathrm{s}$ data rates did not represent error-free scenarios, as confirmed by the BER plot, and their apparent narrow eye aperture was therefore justified. Moreover, in line with the reported OSNR levels at the receiver, a larger noise accompanied the optical levels in Figure 11 with respect to the MZM case. It could be attributed to the usage of an additional EDFA in the RM test, which determined a greater in-band ASE-related noise.

In [17], we already stated that the unexpected results recorded in the MZM case were packaging-dependent, even because error-free transmissions till $5 \mathrm{~Gb} / \mathrm{s}$ were verified from fully electrical measurements made on a standalone driver installed on a testing PCB. As can be seen from the detail in Figure 9, a few bond wires indeed traversed the whole chip and potentially induced undesired couplings between the EIC input/output ports. The eye diagrams in Figure 10 indeed present non-smooth rising/falling edges and multiple traces, which were a clear manifestation of a strong inter-symbol interference (ISI). Instead, the wire bonding arrangement in the RM case appeared clean, and the full $5 \mathrm{~Gb} / \mathrm{s}$ speed rating was achieved for the RM case. This proved the pseudo-differential output stage's correct applicability to single-ended modulators. 


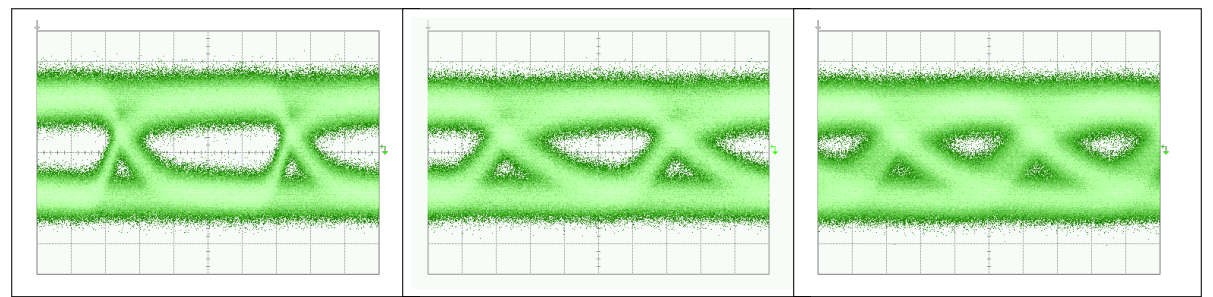

(a) $2 \mathrm{~Gb} / \mathrm{s}$ (c) $5 \mathrm{~Gb} / \mathrm{s}$

Figure 11. Eye diagrams on the PD output port in the RM case study (scale $10 \mathrm{mV} /$ div, condition $\left.P_{\mathrm{pd}}=-6 \mathrm{dBm}\right)$.
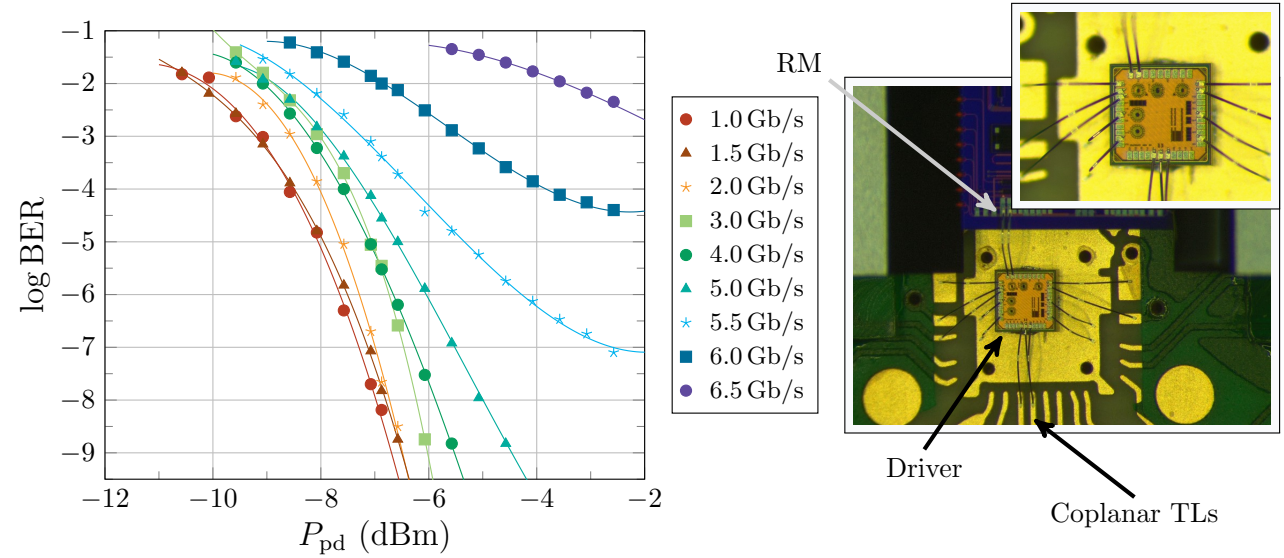

Figure 12. BER performances as a function of the received power $P_{\mathrm{pd}}$ for the RM-based transmission system.

The obtained data rates were relatively low compared to those for data center purposes ( $>25 \mathrm{~Gb} / \mathrm{s}$ [7]) and even with respect to state-of-the-art laser diode-based HEP optical links (5 to $10 \mathrm{~Gb} / \mathrm{s}$ [15]). However, as extensively reported in Section 3, many circuital degrees of freedom (DoFs) have been used to address the radiation hardening requirements, sacrificing speed performances. The proposed circuit had indeed a radiation tolerance compliant with the levels foreseen for the next upgrade of the LHC apparatus, i.e., $8 \mathrm{MGy}$, while the laser diode-oriented gigabit laser driver (GBLD) operation was guaranteed till $1 \mathrm{MGy}$ at most [48]. As already shown in [44], it is important to note that the proposed driver architecture could sustain a $5 \mathrm{~Gb} / \mathrm{s}$ transmission to these extreme radiation levels, though undergoing a reduction in the output voltage swing. The voltage amplitude versus TID trend appeared to be slightly smooth, and only a 30\% decrease was captured at 8 MGy TID [17]. Even though transistor parameters would continue to degrade, it was likely that the driver could operate at even higher doses.

The speed limit of the proposed driver was not entirely imposed by the chosen architecture and the connected SiPh load, but was rather due to the components that surrounded the signal path. A major role was played by the electro-static discharge (ESD) protection circuits deployed around each pad. A building-block solution was chosen because of its recognized radiation hardness despite presenting a capacitance greater than $0.5 \mathrm{pF}$. This large capacitive contribution, together with relatively long wire bonds $(\approx 1 \mathrm{~mm})$, determined a low pass filtering that inevitably affected the overall bandwidth. While being partially compensated by the inductive peaking in the output stage, the input side of the device was completely exposed to this parasitic effect. To enable the scalability of this design approach to higher bandwidths, this downside could be mitigated by embedding compensation or T-coil networks to the ESD diodes, as already done in [48]. Such a technique was not implemented here to save silicon area and because the target was more focused on pushing further the driver operation in terms of radiation tolerance than overcoming state-of-the-art data rates in HEP environments. Moreover, a closer interaction with the packaging facility would help in avoiding assembly flaws as happened for the MZM-based system. A co-design between the handle PCB, the EIC, and the PIC would indeed 
allow estimating the interconnection parasitics earlier in the design process and harness them to make a more efficient and robust system. A re-design of the same driver is already under development to carry out more than $10 \mathrm{~Gb} / \mathrm{s}$ operation with the same architecture.

\section{Conclusions}

Silicon photonics is emerging as a promising technology to realize multi-Gb/s optical links in the detector systems for future HEP experiments. Electronic ASICs are needed to drive photonic modulators and make the electro-optical readout systems fit in compact hybrid modules. In this paper, we addressed the challenge of whether it was possible to lay out a high-speed driver capable of driving a broad variety of SiPh devices, i.e., both differential (LE/TW-MZMs), as well as single-ended (RMs) loads. A pseudo-differential output stage was designed with this purpose and realized in a $65 \mathrm{~nm}$ technology following RHBD techniques. The applicability of the circuit to both LE MZMs and RMs was confirmed by experimental BER testing. A $5 \mathrm{~Gb} / \mathrm{s}$ quasi error-free transmission was achieved in the RM case, while only $1.5 \mathrm{~Gb} / \mathrm{s}$ was obtained with the MZM sample because of a leak in the wire-bonding packaging procedure.

For the next electronic designs, particular attention will be given to the interconnection and ESD circuits' modeling, preserving the required space to implement inductive broad banding techniques in order to achieve higher transmission speeds with the same radiation-tolerance ratings. Additionally, a photonic chip comprising several modulators will be submitted to test the proposed driver in other loading conditions. Each modulator will be also equipped with thermal phase shifters to have more degrees of freedom to set the working point in the experimental characterization. Another perspective work will be the irradiation test of the whole co-packaged SiPh-based system to recreate the target working conditions reliably and further validate its operation in harsh environments.

Author Contributions: Conceptualization, S.C.; methodology, S.C., G.C., S.F., and P.V.; validation, S.C., G.C., and S.F.; investigation, S.C., G.C., and S.F.; resources, G.C., S.F., P.V., and G.M.; data curation, S.C., G.C., and S.F.; writing, original draft preparation, S.C.; writing, review and editing, S.C., G.C., S.F., F.P., and S.S.; visualization, S.C.; supervision, F.P. and S.S.; project administration, F.P. and S.S.; funding acquisition, F.P. and S.S. All authors read and agreed to the published version of the manuscript.

Funding: This research was funded by INFN under the grant PHOS4BRAIN and by University of Pisa/INFN in the framework of the ISHTAR project.

Acknowledgments: The authors would like to acknowledge A. Kraxner, C. Scarcella, J. Troska, and F. Vasey for their support during the work and for providing the photonic integrated circuit, the printed circuit board for hybrid integration, and some electronic building blocks.

Conflicts of Interest: The authors declare no conflict of interest.

\section{References}

1. Backhaus, M. The Upgrade of the CMS Inner Tracker for HL-LHC; Technical Report CMS-CR-2019-011; CERN: Geneva, Switzerland, 2019.

2. Amaral, L.; Dris, S.; Gerardin, A.; Huffman, T.; Issever, C.; Pacheco, A.J.; Jones, M.; Kwan, S.; Lee, S.C.; Liang, Z.; et al. The versatile link, a common project for super-LHC. J. Instrum. 2009, 4, P12003. [CrossRef]

3. Vasey, F.; Hall, D.; Huffman, T.; Kwan, S.; Prosser, A.; Soos, C.; Troska, J.; Weidberg, T.; Xiang, A.; Ye, J. The Versatile Link common project: feasibility report. J. Instrum. 2012, 7, C01075. [CrossRef]

4. Troska, J.; Detraz, S.; El Nasr-Storey, S.S.; Stejskal, P.; Sigaud, C.; Soos, C.; Vasey, F. Radiation Damage Studies of Lasers and Photodiodes for Use in Multi-Gb/s Optical Data Links. IEEE Trans. Nucl. Sci. 2011, 58, 3103-3110. [CrossRef]

5. Mazza, G.; Rivetti, A.; Moreira, P.; Wyllie, K.; Soos, C.; Troska, J.; Gui, P. A radiation tolerant 5 Gb/s Laser Driver in $130 \mathrm{~nm}$ CMOS technology. J. Instrum. 2012, 7, C01052. [CrossRef]

6. Seif El Nasr-Storey, S.; Boeuf, F.; Baudot, C.; Detraz, S.; Fedeli, J.M.; Marris-Morini, D.; Olantera, L.; Pezzullo, G.; Sigaud, C.; Soos, C.; et al. Effect of Radiation on a Mach-Zehnder Interferometer Silicon Modulator for HL-LHC Data Transmission Applications. IEEE Trans. Nucl. Sci. 2015, 62, 329-335. [CrossRef] 
7. Thomson, D.; Zilkie, A.; Bowers, J.E.; Komljenovic, T.; Reed, G.T.; Vivien, L.; Marris-Morini, D.; Cassan, E.; Virot, L.; Fédéli, J.M.; et al. Roadmap on silicon photonics. J. Opt. 2016, 18, 073003. [CrossRef]

8. Oblakowska-Mucha, A. Radiation Hard Silicon Particle Detectors for Phase-II LHC Trackers. J. Instrum. 2017, 12, C02054. [CrossRef]

9. Bogaerts, W.; Chrostowski, L. Silicon Photonics Circuit Design: Methods, Tools and Challenges. Laser Photonics Rev. 2018, 12, 1700237. [CrossRef]

10. Nasr-Storey, S.E.; Détraz, S.; Olanterä, L.; Sigaud, C.; Soós, C.; Pezzullo, G.; Troska, J.; Vasey, F.; Zeiler, M. Neutron and X-ray irradiation of silicon based Mach-Zehnder modulators. J. Instrum. 2015, 10, C03040. [CrossRef]

11. Zeiler, M.; El Nasr-Storey, S.S.; Detraz, S.; Kraxner, A.; Olantera, L.; Scarcella, C.; Sigaud, C.; Soos, C.; Troska, J.; Vasey, F. Radiation Damage in Silicon Photonic Mach-Zehnder Modulators and Photodiodes. IEEE Trans. Nucl. Sci. 2017, 64, 2794-2801. [CrossRef]

12. Kraxner, A.; Detraz, S.; Olantera, L.; Scarcella, C.; Sigaud, C.; Soos, C.; Stile, C.; Troska, J.; Vasey, F. Radiation tolerance enhancement of silicon photonics for HEP applications. Proc. Sci. 2019, 17. [CrossRef]

13. Rahim, A.; Spuesens, T.; Baets, R.; Bogaerts, W. Open-Access Silicon Photonics: Current Status and Emerging Initiatives. Proc. IEEE 2018, 106, 2313-2330. [CrossRef]

14. Pantouvaki, M.; Srinivasan, S.A.; Ban, Y.; De Heyn, P.; Verheyen, P.; Lepage, G.; Chen, H.; De Coster, J.; Golshani, N.; Balakrishnan, S.; et al. Active Components for $50 \mathrm{~Gb} / \mathrm{s}$ NRZ-OOK Optical Interconnects in a Silicon Photonics Platform. J. Lightw. Technol. 2017, 35, 631-638. [CrossRef]

15. Mazza, G.; Tavernier, F.; Moreira, P.; Calvo, D.; De Remigis, P.; Olantera, L.; Soos, C.; Troska, J.; Wyllie, K. High-Speed, Radiation-Tolerant Laser Drivers in $0.13 \mu \mathrm{m}$ CMOS Technology for HEP Applications. IEEE Trans. Nucl. Sci. 2014, 61, 3653-3659. [CrossRef]

16. Zhang, Y.; Schneider, M.; Karnick, D.; Eisenblätter, L.; Kühner, T.; Weber, M. Key building blocks of a silicon photonic integrated transmitter for future detector instrumentation. J. Instrum. 2019, 14, P08021. [CrossRef]

17. Ciarpi, G.; Cammarata, S.; Faralli, S.; Vehla, P.; Magazzù, G.; Palla, F.; Saponara, S. Design, Operation and BER Test of Multi-Gb/s Radiation-Hard Drivers in $65 \mathrm{~nm}$ Technology for Silicon Photonics Optical Modulators. In Applications in Electronics Pervading Industry, Environment and Society; Saponara, S., De Gloria, A., Eds.; Springer International Publishing: Cham, Switzerland, 2020; pp. 11-18.

18. Reed, G.; Mashanovich, G.; Gardes, F.; Thomson, D. Silicon optical modulators [Review]. Nat. Photonics 2010, 4, 518-526. [CrossRef]

19. Nedeljkovic, M.; Soref, R.; Mashanovich, G.Z. Free-Carrier Electrorefraction and Electroabsorption Modulation Predictions for Silicon Over the 1-14- $\mu \mathrm{m}$ Infrared Wavelength Range. IEEE Photonics J. 2011, 3, 1171-1180. [CrossRef]

20. Witzens, J. High-Speed Silicon Photonics Modulators. Proc. IEEE 2018, 106, 2158-2182. [CrossRef]

21. Fresi, F.; Malacarne, A.; Sorianello, V.; Meloni, G.; Velha, P.; Midrio, M.; Toccafondo, V.; Faralli, S.; Romagnoli, M.; Poti, L. Reconfigurable Silicon Photonics Integrated 16-QAM Modulator Driven by Binary Electronics. IEEE J. Select. Top. Quant. Electron. 2016, 22, 334-343. [CrossRef]

22. Bogaerts, W.; De Heyn, P.; Van Vaerenbergh, T.; De Vos, K.; Kumar Selvaraja, S.; Claes, T.; Dumon, P.; Bienstman, P.; Van Thourhout, D.; Baets, R. Silicon microring resonators. Laser Photonics Rev. 2012, 6, 47-73. [CrossRef]

23. Malacarne, A.; Gambini, F.; Faralli, S.; Klamkin, J.; Poti, L. High-Speed Silicon Electro-Optic Microring Modulator for Optical Interconnects. IEEE Photonics Technol. Lett. 2014, 26, 1042-1044. [CrossRef]

24. Van Kerrebrouck, J.; De Keulenaer, T.; Pierco, R.; De Geest, J.; Sinsky, J.H.; Kozicki, B.; Yin, X.; Torfs, G.; Bauwelinck, J. NRZ, Duobinary, or PAM4?: Choosing Among High-Speed Electrical Interconnects. IEEE Microw. Mag. 2019, 20, 24-35. [CrossRef]

25. Xiao, X.; Xu, H.; Li, X.; Hu, Y.; Xiong, K.; Li, Z.; Chu, T.; Yu, Y.; Yu, J. 25 Gbit/s silicon microring modulator based on misalignment-tolerant interleaved PN junctions. Opt. Express 2012, 20, 2507-2515. [CrossRef] [PubMed]

26. Temporiti, E.; Ghilioni, A.; Minoia, G.; Orlandi, P.; Repossi, M.; Baldi, D.; Svelto, F. Insights Into Silicon Photonics Mach-Zehnder-Based Optical Transmitter Architectures. IEEE J. Solid-State Circ. 2016, 51, 3178-3191. [CrossRef] 
27. Zhou, S.; Ta Wu, H.; Sadeghipour, K.; Scarcella, C.; Eason, C.; Rensing, M.; Power, M.J.; Antony, C.; O'Brien, P.; Townsend, P.D.; et al. Optimization of PAM-4 transmitters based on lumped silicon photonic MZMs for high-speed short-reach optical links. Opt. Express 2017, 25, 4312-4325. [CrossRef] [PubMed]

28. Li, K.; Thomson, D.J.; Liu, S.; Meng, F.; Shakoor, A.; Khokhar, A.; Cao, W.; Zhang, W.; Wilson, P.; Reed, G.T. Co-Design of Electronics and Photonics Components for Silicon Photonics Transmitters. In Proceedings of the 2018 European Conference on Optical Communication (ECOC), Rome, Italy, 23-27 September 2018; pp. 1-3. [CrossRef]

29. Razavi, B. Design of Integrated Circuits for Optical Communications; Wiley: Hoboken, NJ, USA, 2012.

30. Li, K.; Thomson, D.J.; Liu, S.; Wilson, P.; Reed, G.T. A 30 Gb/s CMOS driver integrated with silicon photonics MZM. In Proceedings of the 2015 IEEE Radio Frequency Integrated Circuits Symposium (RFIC), Phoenix, AZ, USA, 17-19 May 2015; pp. 311-314.

31. Li, C.; Yu, K.; Rhim, J.; Zhu, K.; Qi, N.; Fiorentino, M.; Pinguet, T.; Peterson, M.; Saxena, V.; Palermo, S. A 3D-Integrated $56 \mathrm{~Gb} / \mathrm{s}$ NRZ/PAM4 Reconfigurable Segmented Mach-Zehnder Modulator-Based Si-Photonics Transmitter. In Proceedings of the 2018 IEEE BiCMOS and Compound Semiconductor Integrated Circuits and Technology Symposium (BCICTS), San Diego, CA, USA, 14-17 October 2018; pp. 32-35. [CrossRef]

32. Rakowski, M.; Ryckaert, J.; Pantouvaki, M.; Yu, H.; Bogaerts, W.; de Meyer, K.; Steyaert, M.; Absil, P.P.; Van Campenhout, J. Low-Power, 10-Gbps 1.5-Vpp differential CMOS driver for a silicon electro-optic ring modulator. In Proceedings of the IEEE 2012 Custom Integrated Circuits Conference, San Jose, CA, USA, 9-12 September 2012, pp. 1-6. [CrossRef]

33. Zhou, S.; Wu, H.; Sadeghipour, K.; Scarcella, C.; Eason, C.; Rensing, M.; Power, M.; Antony, C.; O’Brien, P.; Townsend, P.; et al. Driver circuit for a PAM-4 optical transmitter using $65 \mathrm{~nm}$ CMOS and silicon photonic technologies. Electron. Lett. 2016, 52, 1939-1940. [CrossRef]

34. Cignoli, M.; Minoia, G.; Repossi, M.; Baldi, D.; Ghilioni, A.; Temporiti, E.; Svelto, F. 22.9 A 1310nm 3D-integrated silicon photonics Mach-Zehnder-based transmitter with $275 \mathrm{~mW}$ multistage CMOS driver achieving $6 \mathrm{~dB}$ extinction ratio at $25 \mathrm{~Gb} / \mathrm{s}$. In Proceedings of the 2015 IEEE International Solid-State Circuits Conference-(ISSCC), San Francisco, CA, USA, 22-26 February 2015; pp. 1-3. [CrossRef]

35. Liao, Q.; Qi, N.; Li, M.; Hu, S.; He, J.; Yin, B.; Shi, J.; Liu, J.; Chiang, P.Y.; Xiao, X.; et al. A 50-Gb/s PAM4 Si-Photonic Transmitter With Digital-Assisted Distributed Driver and Integrated CDR in 40-nm CMOS. IEEE J. Solid-State Circ. 2020, 55, 1282-1296. [CrossRef]

36. Faccio, F.; Borghello, G.; Lerario, E.; Fleetwood, D.M.; Schrimpf, R.D.; Gong, H.; Zhang, E.X.; Wang, P.; Michelis, S.; Gerardin, S.; et al. Influence of LDD Spacers and H+ Transport on the Total-Ionizing-Dose Response of 65-nm MOSFETs Irradiated to Ultrahigh Doses. IEEE Trans. Nucl. Sci. 2018, 65, 164-174. [CrossRef]

37. Bonaldo, S.; Mattiazzo, S.; Enz, C.; Baschilotto, A.; Fleetwood, D.M.; Paccagnella, A.; Gerardin, S. Ionizing-Radiation Response and Low-Frequency Noise of 28-nm MOSFETs at Ultra-High Doses. IEEE Trans. Nucl. Sci. 2020. [CrossRef]

38. Bonaldo, S.; Gerardin, S.; Jin, X.; Paccagnella, A.; Faccio, F.; Borghello, G.; Fleetwood, D.M. Charge Buildup and Spatial Distribution of Interface Traps in 65-nm pMOSFETs Irradiated to Ultrahigh Doses. IEEE Trans. Nucl. Sci. 2019, 66, 1574-1583. [CrossRef]

39. Ciarpi, G.; Saponara, S.; Magazzù, G.; Palla, F. Radiation Hardness by Design Techniques for 1 Grad TID Rad-Hard Systems in $65 \mathrm{~nm}$ Standard CMOS Technologies. In Applications in Electronics Pervading Industry, Environment and Society; Saponara, S., De Gloria, A., Eds.; Springer International Publishing: Cham, Switzerland, 2019; pp. 269-276.

40. Snoeys, W.J.; Gutierrez, T.A.P.; Anelli, G. A new NMOS layout structure for radiation tolerance. IEEE Trans. Nucl. Sci. 2002, 49, 1829-1833. [CrossRef]

41. Sedighi, B.; Christoph Scheytt, J. 40 Gb/s VCSEL driver IC with a new output current and pre-emphasis adjustment method. In Proceedings of the 2012 IEEE/MTT-S International Microwave Symposium Digest, Montreal, QC, Canada, 17-22 June 2012; pp. 1-3. [CrossRef]

42. Zandieh, A.; Schvan, P.; Voinigescu, S.P. Linear Large-Swing Push-Pull SiGe BiCMOS Drivers for Silicon Photonics Modulators. IEEE Trans. Microw. Theory Tech. 2017, 65, 5355-5366. [CrossRef]

43. Jeong, G.S.; Bae, W.; Jeong, D.K. Review of CMOS Integrated Circuit Technologies for High-Speed Photo-Detection. Sensors 2017, 17, 1962. [CrossRef] [PubMed] 
44. Ciarpi, G.; Magazzù, G.; Palla, F.; Saponara, S. Design, Implementation, and Experimental Verification of 5 Gbps, 800 Mrad TID and SEU-Tolerant Optical Modulators Drivers. IEEE Trans. Circ. Syst. I Regul. Pap. 2020, 67, 829-838. [CrossRef]

45. Zeiler, M.; Detraz, S.; Olantera, L.; Pezzullo, G.; Nasr-Storey, S.S.E.; Sigaud, C.; Soos, C.; Troska, J.; Vasey, F. Design of Si-photonic structures to evaluate their radiation hardness dependence on design parameters. J. Instrum. 2016, 11, C01040. [CrossRef]

46. Carroll, L.; Lee, J.S.; Scarcella, C.; Gradkowski, K.; Duperron, M.; Lu, H.; Zhao, Y.; Eason, C.; Morrissey, P.; Rensing, M.; et al.. Photonic Packaging: Transforming Silicon Photonic Integrated Circuits into Photonic Devices. Appl. Sci. 2016, 6, 426. [CrossRef]

47. Hui, R.; O'Sullivan, M. Optical System Performance Measurements. In Fiber Optic Measurement Techniques; Hui, R.; O'Sullivan, M., Eds.; Academic Press: New York, NY, USA, 2009; Chapter 5, pp. 481-630. [CrossRef]

48. Mazza, G.; Tavernier, F.; Moreira, P.; Rivetti, A.; Soos, C.; Troska, J.; Wyllie, K. The GBLD: A radiation tolerant laser driver for high energy physics applications. J. Instrum. 2013, 8, C01033. [CrossRef]

(C) 2020 by the authors. Licensee MDPI, Basel, Switzerland. This article is an open access article distributed under the terms and conditions of the Creative Commons Attribution (CC BY) license (http://creativecommons.org/licenses/by/4.0/). 